(C) Copyright 1999 IEEE. IEEE $49^{\text {th }}$ Vehicular Technology Conference (VTC'99), May, 16-19, 1999, Houston, Texas

Personal use of this material is permitted. However, permission to reprint/republish this material for advertising or promotional purposes or for creating new collective works for resale or redistribution to servers or lists, or to reuse any copyrighted component of this work in other works must be obtained from the IEEE. 


\title{
PERFORMANCE ANALYSIS OF SEMI-BLIND SIGNAL SEPARATION AND DETECTION FOR SMART ANTENNAS
}

\author{
Juha Laurila, Klaus Kopsa, Robert Schürhuber, and Ernst Bonek \\ Technische Universität Wien, \\ Institut für Nachrichtentechnik und Hochfrequenztechnik \\ Gußhausstrasse 25/389, A-1040 Wien, Austria \\ Tel. +43-1-58801389 85, Fax. +43-1-5870 583 \\ e-mail: juha.laurila@nt.tuwien.ac.at
}

\begin{abstract}
We describe a semi-blind algorithm for smart antennas that, in addition to structural signal properties, utilises user identifiers that are available in existing cellular systems. Assuming two co-channel users in a GSM-like TDMA system and a Rayleigh fading channel with finite angular spread, we obtained a $\mathrm{BER}<10^{-3}$ with only 2-3 antennas. In this paper we show the performance of the algorithm in several different simulation scenarios also including array imperfections and angularly non-separable sources.
\end{abstract}

\section{INTRODUCTION}

Smart antenna processing for cellular mobile radio has traditionally focused on temporal or spatial reference techniques. Recently, more attention has been paid to blind source separation and signal detection without directional information. With these techniques, the channel response matrix which maps the transmitted signals to the received array data samples, can be identified without the aid of known bit sequences. Combining equalisation and detection, these methods yield the estimated symbol sequences directly.

From a mobile communications point of view we see several benefits that motivate the use of blind estimation methods:

- Neither limited angular spread nor angular separability are necessary

- Robustness against receiver imperfections - relaxed requirements for array calibration

- The incoming signals can be unsynchronised

- Reduced need for overhead information additional capacity increase.

In the present paper we describe a semi-blind algorithm, employing the user identification fields to initialise the estimation. The reason to focus on the semi-blind method instead of totally blind is twofold. First, in a SDMA (Space Division Multiple Access) system, where several users are served simultaneously in the same traffic channel, some kind of known information must be included in the transmitted signals for the purpose of user identification. Second, the frame structures of all current TDMA-based mobile systems contain training sequences for channel estimation. Thus, it is reasonable to utilise this known information already during the estimation process, not only after detection of the symbol vectors.

Our semi-blind estimation method yields directly the desired symbol vectors by performing three tasks: joint space-time equalisation, separation, and detection of the multiple oversampled co-channel digital signals. The estimation process consists of two parts. First, we estimate the basis for the row span of the received data matrix. After that these obtained basis vectors are projected to the finite alphabet (FA) constellation using our DILSF algorithm. Between these two steps we initialise the FA projections with user identification fields. This leads to fast convergence and an increased robustness of DILSF. Figure 1 demonstrates the structure of this algorithm.

Our estimation technique uses two structural signal properties: The fixed symbol rate allows the factorisation of the received data onto the channel response and signal matrices by means of their special structures. Utilisation of the finite alphabet (i.e. the limited number of modulation symbols) together with the first property allows solving of the FIR-MIMO (Finite Impulse Response, Multiple Input, Multiple Output) problem.

The aim of this paper is to demonstrate the performance of algorithm in different simulation scenarios. The interested reader can find more details about the algorithm itself in (1),(2) and references therein.

We use the following notation throughout the paper. $A^{\#}$ and $\|A\|$ denote the Moore-Penrose pseudo-inverse and 
the Frobenius norm of the matrix $A$, respectively. The notation $\operatorname{row}(A)$ denotes the row span of $A$.

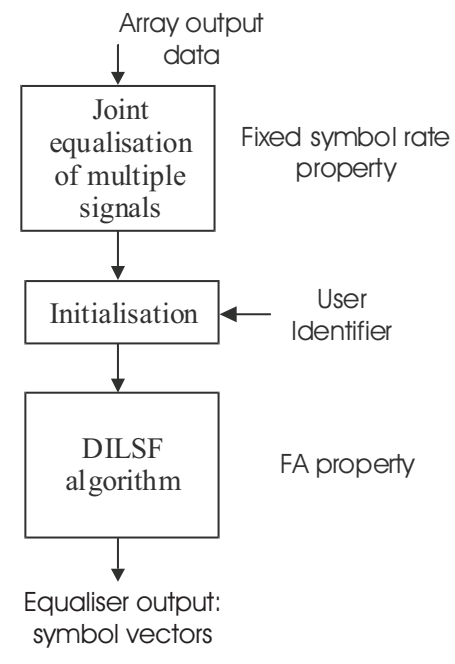

Fig.1. Semi-blind detection

\section{PARTS OF THE ALGORITHM}

\section{Joint Space-Time Equalisation}

Joint space-time equalisation based on subspace estimation is the first part of the detection process (first block in Fig.2.).

An array of $M$ antennas receives $d$ digital signals $s_{l}(t), \ldots, s_{d}(t)$ transmitted over independent finite impulse response channels which have a maximal length of $L$ symbols. Each antenna element is oversampled with a rate $P$ and the data matrix $X$ is constructed by collecting data over $N$ symbol periods. The $n$-th column of this matrix contains $M \cdot P$ samples received during the transmission of the $n$-th symbol. Our blind estimation problem is to factorise the received data onto the matrices corresponding to space-time channel, $H$, and estimated data, $S$, i.e. we solve equation $X=H S$.

The estimation is based on the equality of the row spans of the data and symbol matrices, i.e. $\operatorname{row}(X)=\operatorname{row}(S)$, which requires the channel response matrix $H$ to have full rank. To guarantee this property with reasonable parameter settings, we create an extended data matrix, $\boldsymbol{x}$, by left-shifting and stacking the original data blocks $m$ times. This stacking parameter $m$ can be regarded as the length of the time-domain equaliser. For the extended data matrix the blind estimation problem can be written, $x=75$.

The first step is to estimate the orthonormal basis of the row span of $\boldsymbol{X}$, which is equal to that of $\boldsymbol{S}$ assuming that the full-rank property of the channel matrix is fulfilled. The optimal way to do this is to perform singular value decomposition (SVD) of the data matrix, $\boldsymbol{X}=U \Sigma V(3)$. The first $d_{\mathcal{x}}$ rows of $V$ form an orthonormal basis for the row span of $\boldsymbol{x}$, where the rank value $d_{\boldsymbol{x}}$, satisfies the equation $d_{\mathcal{X}}=d(L+m-1)$. If the number of incoming signals, $d$, is not known, it can be estimated using this property. Instead of SVD also suboptimal but computationally cheaper subspace tracking algorithms (4) can be employed for subspace estimation. We discuss this trade-off between complexity and performance in (1).

The dimensionality of the basis, $\delta$, can be decreased by introducing subspace intersections (2). In case of full intersections, the number of remaining basis vectors can be decreased to the number of incoming signals, i.e. $\delta=d$.

\section{Finite Alphabet Projections}

After subspace estimation, we have to define which linear combination of these basis vectors gives a finite alphabet structure. For this purpose we can employ an iterative least-squares technique (5),(9). The performance of the projection algorithms is crucially dependent on the accuracy of the initialisation, especially when GMSK modulation is used. In (6) and (7) the analytical constant modulus algorithm (ACMA) (8) improves the trivial initialisation before the FA projections.

In contrast, we propose to employ user identifiers to initialise the iterative projections. Thus, we can omit the ACMA part even with non-linear GMSK modulation. In addition to the reduced computational burden, this improves the robustness of the estimation.

\section{DILSF Algorithm}

We call our projection algorithm Decoupled Iterative Least Squares with Subspace Fitting, DILSF. It combines the ideas of the DWILSP (Decoupled Weighted Iterative Least Squares with Projections) (9) and the ILSF (Iterative Least Squares with Subspace Fitting) algorithms (2). Instead of a simultaneous estimation of all symbol vectors, this approach makes the projections between the FA constellation and the obtained subspace separately for each user, which has desirable properties.

The goal of the alternating least squares projections is to find symbol vectors $s_{i}$ (belonging to the FA constellation $\Omega$ ) and corresponding projection vectors $t_{i}$, so that the mean square error $\left\|s_{i}-t_{i} \cdot Y\right\|^{2}$ is minimised for each user $i$. The input matrix $Y$ contains $\delta$ orthonormal basis vectors from the preceding part of the algorithm. During the iterations we need only the 
pseudo-inverse of the input matrix $Y$, which remains constant over all iterations.

The initialisation of the fitting vectors, $t_{i}^{(0)}$, by user identification fields ensures convergence to the global minimum. The correct initialisation block $Y_{U I D}$ of the input matrix is selected by correlating basis vectors and user identifier. We obtain then the initialisation value, $t_{i}^{(0)}$, as a least-squares solution using block $Y_{U I D}$ and the identifier of user $i$. We continue iterations until convergence is reached and after that we estimate the next desired signal. The algorithm converges very fast; in most simulations the correct symbol vectors were obtained already after the first iteration round. Figure 2 shows the $\mathrm{CDF}$ for the number of required iterations.

TABLE 1: SOLVING $\min _{s, t ; s \in F A}\left\|s_{i}-t_{i} \cdot Y\right\|^{2} \quad$ FOR EACH DESIRED USER $i$ WITH DILSF-ALGORITHM

\section{for $i=1: d$}

initialise $t_{i}$ with user identifier: $t_{i}^{(0)}=S_{U I D, i} \cdot Y_{U I D}{ }^{\#}$ for $k=1,2, \ldots$

$$
\begin{aligned}
& \text { i) } s_{i}^{(k)}=\operatorname{proj}_{\Omega}\left[t_{i}^{(k-1)} \cdot Y\right] \\
& \text { ii) } t_{i}^{(k)}=s_{i}^{(k)} \cdot Y^{\#} \\
& \text { * repeat until }\left(t^{(k)}, s^{(k)}\right)=\left(t^{(k-1)}, s^{(k-1)}\right)
\end{aligned}
$$

* repeat for all desired signals

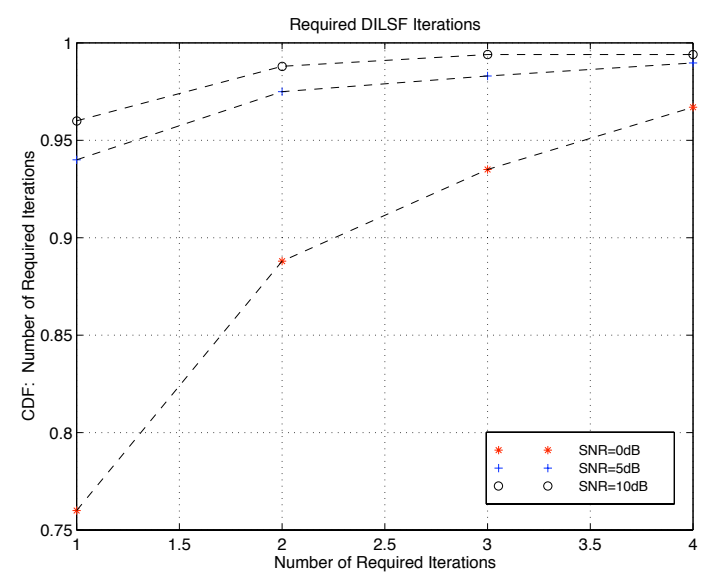

Fig.2. CDF for number of required iterations for convergence

\section{SIMULATIONS}

\section{Channel Model}

We carried out the simulations using the directional Geometry-based Stochastic Channel Model, GSCM (10),(11). In the model the users are surrounded by local scattering areas. Environments causing a larger delay and angular spread are modelled by additional scattering areas at random positions. Physically these far scatterers correspond to the signal echoes e.g. from high-rise buildings or nearby mountains.

Appropriate parameter selection allows simulations of various propagation environments. In this paper we considered the urban environment with base station antennas above rooftop level. This parameter selection gives an averaged angular spread of about 3 degrees around each nominal direction-of-arrival (DOA).

\section{Air Interface}

Our simulations refer to the GSM radio interface (12) with slightly modified training sequences (improved cross-correlation properties (13)) and use a linear approximation of GMSK (14). Note, however, that the same estimation principle is also applicable to any other system fulfilling the required signal properties.

The results of our simulations are raw bit error rates (BER) without error correction coding. We assumed two co-channel SDMA users with ideal slow power control. This means that both users had equal mean powers at the base station but the instantaneous values varied due to the Rayleigh fading. The signal-to-noise ratio values were defined by the received mean power values averaged over a large number of random channel situations. Table 2 shows the used parameter selection of the algorithm.

TABLE 2: SIMULATION PARAMETERS

$\begin{array}{ll}N=\text { Number of Snapshots } & 90 \\ P=\text { Oversampling Rate } & 2 \\ M=\text { Number of Antennas } & 2,3,4,6,8 \\ m=\text { Equaliser Length (stacking) } & 5\end{array}$

\section{Results}

Figure 3 demonstrates the BER performance of the above-described technique when the number of antenna elements in the $\lambda / 2$ spaced uniform linear array (ULA) was varied. The array with more antennas leads to larger data matrices and thus improves the accuracy of subspace estimation.

Figure 4 shows that also a small number of antenna elements provides appropriate performance. In this simulation we decreased the number of antennas but the element spacing was increased to yield a constant array aperture. Thus, the spacing was $20 \lambda, 10 \lambda$ and $7 \lambda$ corresponding to the array sizes of $M=2, M=3$ and $M=4$ elements, respectively (the overall length of the array was about $20 \lambda$ ). The larger distance reduces the correlation between fading signals at the independent elements and thus improves performance compared to the $\lambda / 2$ spaced situation. 
Figure 5 demonstrates this behaviour more accurately by showing the BER with three and four antennas as a function of inter-element spacing. With considered channel scenarios the correlation coefficient was already so small with the spacing of $5 \lambda$ that larger distances did not improve performance further.

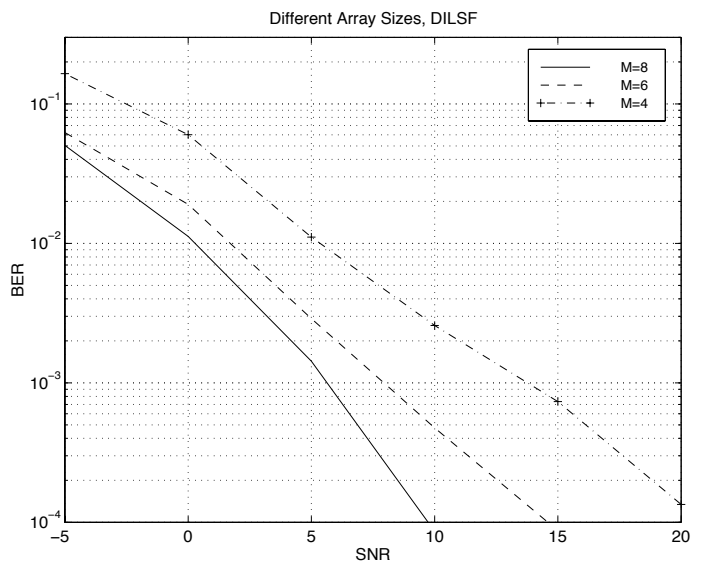

Fig. 3. BER with uniform linear array, $\lambda / 2$ spacing, $M=4,6,8$

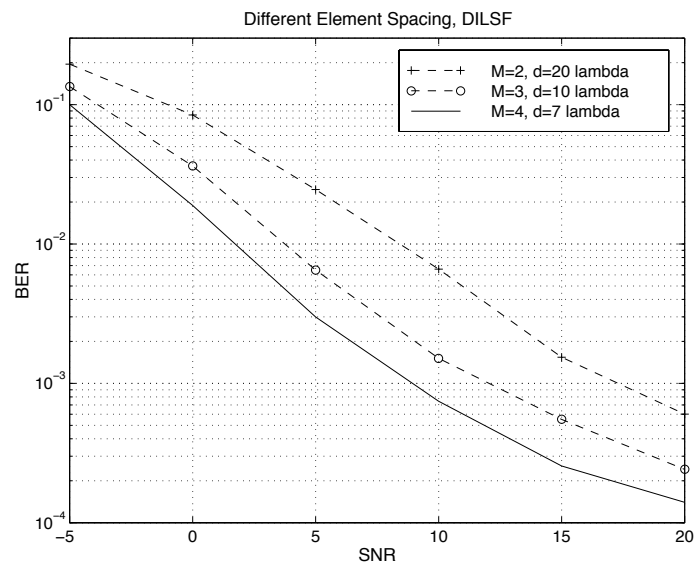

Fig.4. BER with different element spacing, $M=2,3,4$

Figure 6 shows the performance when the power of the interferer was varied, whereas the signal and noise powers remained constant (fixed SNR). This simulation scenario demonstrates that the system is robust against interference and the performance of the separation and detection algorithm is purely noise-limited.

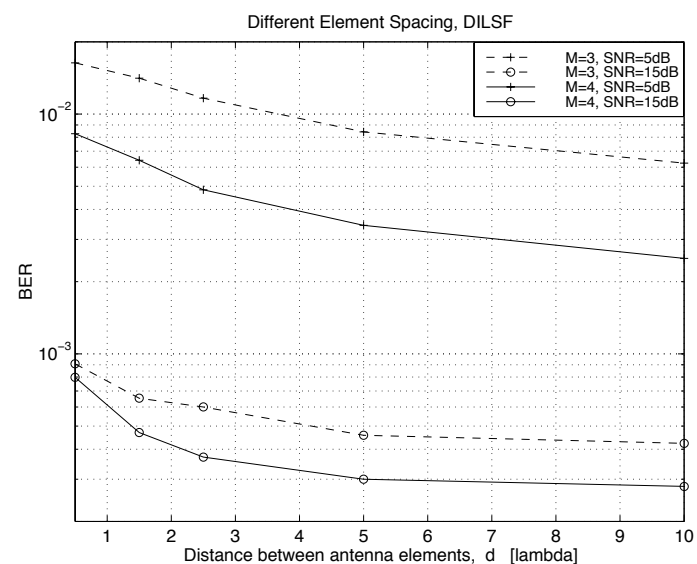

Fig.5. BER as a function of inter-element spacing, $M=3,4$

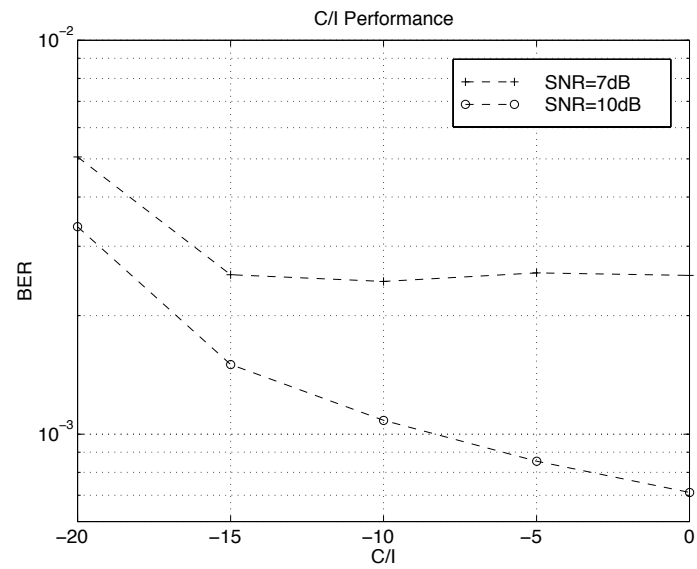

Fig. 6. BER as a function of $\mathrm{C} / \mathrm{I}, M=3, d=10 \lambda$

The performance shown in these figures is very promising especially concerning RF hardware realisation. Results show that already a small number of elements $(M=2-3)$ with two times oversampling gives sufficient performance. Increasing the number of independent samples by using an array with more elements improves performance further.

\section{Special Simulation Scenarios}

\section{Angular separability:}

With traditional DOA-estimation algorithms the detection of different signals is possible if they are separable in the angular domain. Our algorithm is based on the estimation of the unknown channel response matrix, which enables also separation of angularly overlapping signals provided that their channel responses are different.

To demonstrate this property we created the following channel scenario. Instead of random positioning of the 
mobiles we placed both users close to one another (DOA difference $2^{\circ}$, same MS-BS distance). Additionally we used the same scattering points for both users (only local scatterers were present). Thus, in this worst case scenario (Fig. 7.) all received signal components transmitted by two co-channel users were propagating via the same scattering points.

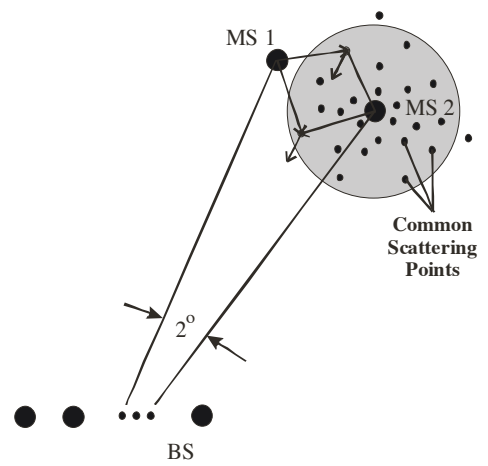

Fig. 7. Common multipath scenario

The different channel response matrices allowed signal separation by our algorithm. Figure 8 shows the BER performance of this scenario as the broken line for a $\lambda / 2$ array with 6 elements. The solid line shows the situation in which we used independent far scattering areas for both users, but still common local scatterers. The figure shows that these independent multipath components increased the diverseness of the channel response matrices, and the BER curve approaches the corresponding simulation with the normal channel model (Fig. 3).

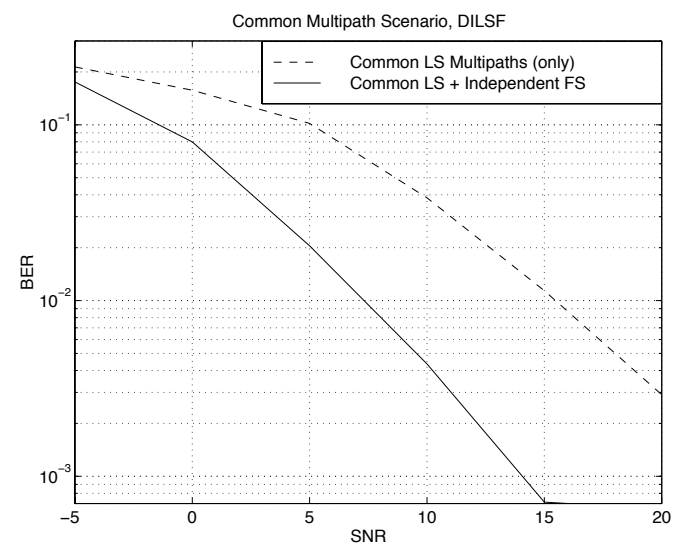

Fig. 8. BER with common multipath scenario, $\lambda / 2$ spacing, $M=6$

\section{Effect of Array Imperfections:}

One benefit of blind techniques is the robustness against array imperfections, because these methods are not relying on a known array manifold. We demonstrated this by multiplying the received data samples with an error model containing hardware non- idealities. Our error model included all the important deviations from ideal hardware like receiver gain and phase imbalances, mutual coupling, oscillator drifts, I/Q imbalances, quantisation noise and cable length differences.

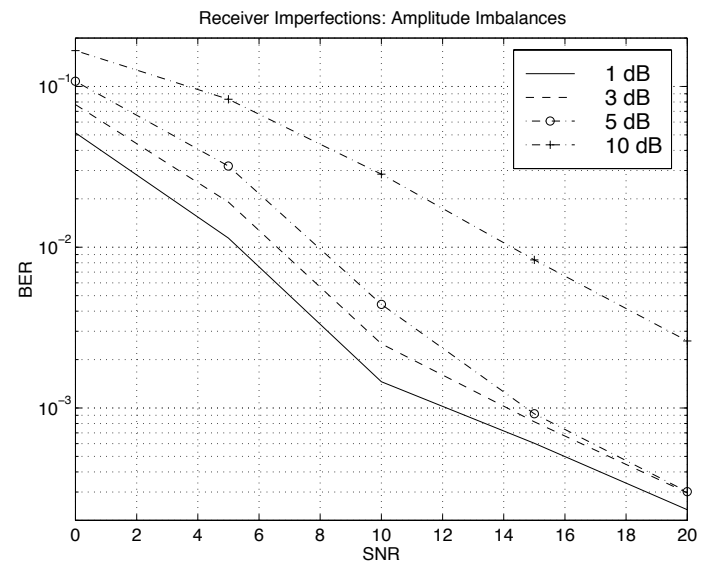

Fig. 9. BER with magnitude imbalances between receiver trains, $\lambda / 2$ spacing, $M=6$

We performed comparative simulations (15) between spatial reference techniques and the algorithm described in this paper. Our results show that SR techniques suffer from imperfections disturbing array manifold structure whereas the semi-blind technique was much more robust. Our algorithm was insensitive against all errors affecting the phase of the received signal, because the phase errors can be hidden in the estimated channel impulse responses. The only imperfection considered that had an effect on the semi-blind receiver was amplitude imbalances between different receiver trains. In this simulation the received samples at antenna outputs were multiplied by different magnitude factors taken from a Gaussian distribution and new values were randomly selected every tenth simulation round. However, this impairment effect was only observed with strong imbalance values exceeding a mean value of $5 \mathrm{~dB}$ (Fig. 9). The reason for this behaviour is that the singular vectors spanning the required subspace are disturbed by multiplicative magnitude factors.

\section{SUMMARY AND CONCLUSIONS}

In this paper we presented a semi-blind estimation principle. First we perform joint space-time equalisation for all incoming signals and estimate a basis for the row span of the data matrix. After that we separate the signals by using the finite alphabet constellation (FA). We also described a method for the required projections, called DILSF (Decoupled Iterative Least Squares with Subspace Fitting) algorithm. This 
technique is based on the decoupled iterative leastsquares projections between obtained subspace and finite alphabet constellation. It requires only matrixvector multiplications during the iteration rounds, which leads to savings in computational complexity. For the initialisation of these projections we use the system specific user identification fields included in the slot structure. The quality of such an initialisation leads to the small number of required iterations before convergence.

We evaluated the performance of the proposed algorithm by simulations with a realistic directional channel model using different array structures. Our results show that increasing the distance between antenna elements improves performance because of the diminished correlation of the signals in the independent sensors. This enables signal separation and detection also with small number of antennas, leading to reduced dimensions of the data matrices and less stringent hardware requirements.

We also treated angularly non-separable signal sources and non-ideal receiver hardware. We obtained excellent bit error rate performance also in case when signals were not separable in angle. By comparative simulations with spatial reference techniques we demonstrated that our semi-blind detector is much more robust against various hardware imperfections.

Acknowledgement: This work was supported by Austrian Fonds zur Förderung der wissenschaftlichen Forschung under Project P12147-ÖMA. Authors thank Andreas F. Molisch and Alexander Kuchar for useful discussions.

\section{REFERENCES}

(1) J. Laurila, K. Kopsa, R. Schürhuber, and E. Bonek, Semi-Blind Separation and Detection of Co-Channel Signals. IEEE International Conference on Communications (ICC '99), Vancouver, Canada, June 6-10, 1999, in press

(2) A-J. van der Veen, S. Talwar, A. Paulraj, A Subspace Approach to Blind Space-Time Signal Processing for Wireless Communications Systems. IEEE Trans. Signal Proc., vol.45, pp. 173-190, Jan. 1997

(3) G. Golub, C.F. Van Loan, Matrix Computations, $2^{\text {nd }} \mathrm{Ed}, 1989,642 \mathrm{p}$.

(4) B. Yang, Projection Approximation Subspace Tracking. IEEE Trans. Signal Proc., vol.43, pp. 95-107, Jan. 1995
(5) S. Talwar, M. Viberg, A. Paulraj, Blind Separation of Synchronous Co-Channel Digital Signals Using an Antenna Array - Part I: Algorithms. IEEE Trans. on Signal Proc., vol.44, pp. 1184-1197, May 1996

(6) A-J. van der Veen, A. Paulraj, Singular Value Analysis of Time-Space Equalization in the GSM Mobile System. Proc. IEEE ICASSP '96. May 1996, Atlanta, GA. pp. 1073-1076

(7) J. Laurila, E. Bonek, SDMA Using Blind Adaptation, ACTS Mobile Communication Summit. Aalborg, Denmark. October 7-10, 1997. pp. 314-319

(8) A.-J. van der Veen, A. Paulraj, An Analytical Constant Modulus Algorithm. IEEE Trans. on Signal Proc., vol.44, pp. 1136-1155, May 1996

(9) P. Pelin, Spatial Diversity Receivers for Base Station Antenna Arrays. Licentiate Thesis, Chalmers University of Technology, Göteborg, Sweden, May 1997, 72 p.

(10) J. Fuhl, A.F. Molisch, E. Bonek, A Unified Channel Model for Mobile Radio Systems with Smart Antennas. IEE Proc. - Radar, Sonar and Navigation: Special Issue on Antenna Array Processing Techniques, vol.145, Feb. 1998, pp. 32-41

(11) A.F. Molisch, J. Laurila, A. Kuchar, R. Schmalenberger, Test scenarios for mobile radio systems with adaptive antennas. First COST 252/259 Joint Workshop, Bradford, UK, April 21-22, 1998, pp. $162-170$

(12) M. Mouly, P. Pautet, The GSM System for Mobile Communications, 1992, $701 \mathrm{p}$.

(13) J. Fuhl, Smart Antennas for Second and Third Generation Mobile Communications Systems. Doctoral Dissertation. INTHFT, TU Wien. Vienna, Austria. 1997. 305 p.

(14) P.A. Laurent, Exact and Approximate Construction of Digital Phase Modulations by Superposition of Amplitude Modulated Pulses (AMP). IEEE Trans. on Comm., vol.34, pp. 150-160, Feb. 1986

(15) R. Schürhuber, Receiver Imperfections and Calibration of Adaptive Antennas, M.Sc. Thesis, INTHF, Technische Universität Wien. Vienna, Austria. 1998, 98 p. 